\title{
Upper and lower solutions satisfying the inverse inequality
}

\author{
by IRENA RACHŮNKOVÁ (Olomouc)
}

\begin{abstract}
We consider multipoint and two-point BVPs for second order ordinary differential equations with a Carathéodory right hand side. We prove the existence of solutions provided there exist upper and lower solutions of the BVP and the upper solution is less than the lower one.
\end{abstract}

1. Introduction. In this paper we consider the four-point boundary value problem

$$
\begin{gathered}
x^{\prime \prime}=f\left(t, x, x^{\prime}\right), \\
x(a)=x(c), \quad x(d)=x(b),
\end{gathered}
$$

where $a, b, c, d \in \mathbb{R}, a<c \leq d<b, J=[a, b]$ and $f: J \times \mathbb{R}^{2} \rightarrow \mathbb{R}$ is a function satisfying the Carathéodory conditions. We prove an existence result for (1), (2) under the assumption that there exist upper and lower solutions of (1), (2) which fulfil the inverse inequality, i.e. the upper solution is less than the lower one (Theorem 1 ).

Since the four-point conditions (2) can be considered as an approximation of the Neumann conditions

$$
x^{\prime}(a)=0, \quad x^{\prime}(b)=0,
$$

our result is valid for problem (1), (3) as well. Moreover, one of the sign conditions for $f$ may be omitted (Theorem 5 ). The same approach can be used for the periodic conditions

$$
x(a)=x(b), \quad x^{\prime}(a)=x^{\prime}(b)
$$

(Theorem 5).

1991 Mathematics Subject Classification: 34B10, 34B15.

Key words and phrases: existence, multipoint and two-point BVP, upper and lower solutions, topological degree.

Research supported in part by the Grant Agency of Czech Republic, Grant no. 301/93/2311 and in part by Ministry of Education of Czech Republic, FRVŠ, Grant no. 0524/1995. 
Let us recall that $\sigma_{1}, \sigma_{2} \in \mathbf{A} \mathbf{C}^{1}(J)$ (i.e. with absolutely continuous first derivatives on $J)$ are lower and upper solutions of $(1),(k)$ if for a.e. $t \in J$,

$$
\sigma_{1}^{\prime \prime}(t) \geq f\left(t, \sigma_{1}, \sigma_{1}^{\prime}\right), \quad \sigma_{2}^{\prime \prime}(t) \leq f\left(t, \sigma_{2}, \sigma_{2}^{\prime}\right),
$$

and for $k=2$,

$$
\begin{array}{ll}
\sigma_{1}(c) \geq \sigma_{1}(a), & \sigma_{1}(b) \leq \sigma_{1}(d), \\
\sigma_{2}(c) \leq \sigma_{2}(a), & \sigma_{2}(b) \geq \sigma_{2}(d)
\end{array}
$$

for $k=3$,

for $k=4$,

$$
\begin{array}{ll}
\sigma_{1}^{\prime}(a) \geq 0, & \sigma_{1}^{\prime}(b) \leq 0 \\
\sigma_{2}^{\prime}(a) \leq 0, & \sigma_{2}^{\prime}(b) \geq 0
\end{array}
$$

$$
\begin{array}{ll}
\sigma_{1}(b)=\sigma_{1}(a), & \sigma_{1}^{\prime}(b) \leq \sigma_{1}^{\prime}(a), \\
\sigma_{2}(b)=\sigma_{2}(a), & \sigma_{2}^{\prime}(b) \geq \sigma_{2}^{\prime}(a) .
\end{array}
$$

Under the classical assumption that

$$
\sigma_{1}(t) \leq \sigma_{2}(t) \quad \text { for all } t \in J
$$

the existence of solutions of various second order boundary value problems has been proved by many authors. We can refer for example to [1], [3], [5]-[7], [17]. For the periodic problem with a Carathéodory right hand side $f$ and a generalized Nagumo condition see [4] or [12].

Here, we investigate the case where $\sigma_{1}, \sigma_{2}$ satisfy the opposite ordering condition

$$
\sigma_{2}(t) \leq \sigma_{1}(t) \quad \text { for all } t \in J
$$

Conditions (5) and (7) are satisfied for example if

$$
\limsup _{|x| \rightarrow \infty} f(t, x, 0) / x<0,
$$

uniformly for a.e. $t \in J$. We can see that (8) yields the existence of constants $r_{1}>0$ and $r_{2}<0$ such that $f\left(t, r_{1}, 0\right) \leq 0, f\left(t, r_{2}, 0\right) \geq 0$ for a.e. $t \in J$. So, we put $\sigma_{1}(t) \equiv r_{1}, \sigma_{2}(t) \equiv r_{2}$ and get lower and upper solutions for problem (1), $(k), k \in\{2,3,4\}$. On the other hand, condition (8) expresses the fact that for large $|x|$ the nonlinearity $f(t, x, 0) / x$ lies on the side of the spectrum of the linear differential operator $\mathbf{L}: x \mapsto x^{\prime \prime}$ acting on the space of functions belonging to $\mathbb{A} C^{1}(J)$ and satisfying $(k), k \in\{2,3,4\}$. This means that the nonlinearity $f(t, x, 0) / x$ could interact with higher eigenvalues of $\mathbb{L}$. This situation was considered e.g. in [2], [9]-[11], [16], [18], mainly for periodic or Dirichlet boundary conditions and for special differential equations like the Liénard or Rayleigh equations.

Our approach is quite different and we present other types of conditions which guarantee the existence of solutions of $(1),(k), k \in\{2,3,4\}$, provided 
(7) is valid. We do not impose growth restrictions on $f$ with respect to $x$ or $x^{\prime}$ but we need some sign conditions for $f$.

\section{Main results}

THEOREM 1. Let $\sigma_{1}$ be a lower solution and $\sigma_{2}$ an upper solution of (1), (2), $\sigma_{1}^{\prime \prime}, \sigma_{2}^{\prime \prime} \in \mathbf{L}_{\infty}(J)$ and let (7) be satisfied. Suppose that there exist real numbers $R_{1}, R_{2}, R_{3}, R_{4}$ such that $R_{1} \neq R_{3}, R_{2} \neq R_{4}, R_{1} \leq \sigma_{i}^{\prime}(t) \leq R_{2}$, $R_{3} \leq \sigma_{i}^{\prime}(t) \leq R_{4}$ for each $t \in J, i=1,2$, and for a.e. $t \in J$,

$$
\begin{aligned}
& f\left(t, x, \sigma_{2}^{\prime}\right) \geq \sigma_{2}^{\prime \prime} \quad \text { for all } x \in\left[c_{1}, \sigma_{2}(t)\right], \\
& f\left(t, x, \sigma_{1}^{\prime}\right) \leq \sigma_{1}^{\prime \prime} \quad \text { for all } x \in\left[\sigma_{1}(t), c_{2}\right],
\end{aligned}
$$

and for all $x \in\left[c_{1}, c_{2}\right]$,

$$
\begin{aligned}
& f\left(t, x, R_{1}\right) \leq 0, \quad f\left(t, x, R_{2}\right) \geq 0 \quad \text { for a.e. } t \in J, \\
& f\left(t, x, R_{3}\right) \geq 0, \quad f\left(t, x, R_{4}\right) \leq 0 \quad \text { for a.e. } t \in[d, b],
\end{aligned}
$$

where $c_{1}=(b-a) L_{1}+\min \left\{\sigma_{2}(t): t \in J\right\}, c_{2}=(b-a) L_{2}+\max \left\{\sigma_{1}(t): t \in J\right\}$, $L_{1}=\min \left\{R_{1}, R_{3}\right\}$ and $L_{2}=\max \left\{R_{2}, R_{4}\right\}$. Then problem (1), (2) has at least one solution $u$ with

$$
\sigma_{2}\left(t_{u}\right) \leq u\left(t_{u}\right) \leq \sigma_{1}\left(t_{u}\right) \quad \text { for some } t_{u} \in J .
$$

COROLlary 2. Let $f$ be nonincreasing in the second variable $x$. Then, in Theorem 1, conditions (9), (10) can be omitted and (11), (12) can be replaced by

$$
\begin{aligned}
& f\left(t, c_{1}, R_{1}\right) \leq 0, \quad f\left(t, c_{2}, R_{2}\right) \geq 0 \quad \text { for a.e. } t \in J, \\
& f\left(t, c_{2}, R_{3}\right) \geq 0, \quad f\left(t, c_{1}, R_{4}\right) \leq 0 \quad \text { for a.e. } t \in[d, b] .
\end{aligned}
$$

EXEMPLE 3 . The function $f(t, x, y)=x-x^{3}+\cos 2 \pi t+\alpha y\left(y^{2}-1\right)$ satisfies the conditions of Theorem 1 for sufficiently large $\alpha$. If we choose $J=[0,1]$, we can put $\sigma_{1}=1.35, \sigma_{2}=-1.35, R_{1}=-0.4, R_{2}=0.4, R_{3}=-1.1$, $R_{4}=1.1$ and $|\alpha| \geq 60$.

EXEMPLE 4. The function $f(t, x, y)=-x+\sin t+\alpha \sin y$ satisfies the conditions of Corollary 2 for sufficiently large $\alpha$. If we choose $J=[a, b]$, we can put $\sigma_{1}=1, \sigma_{2}=-1, R_{1}=-\pi / 2, R_{2}=\pi / 2, R_{3}=-3 \pi / 2, R_{4}=-3 \pi / 2$, $|\alpha|>3 \pi(b-a) / 2+1$.

THEOREM 5. Let $\sigma_{1}$ be a lower solution and $\sigma_{2}$ an upper solution of (1), (k), $k \in\{3,4\}$, with $\sigma_{1}^{\prime \prime}, \sigma_{2}^{\prime \prime} \in \mathbf{L}_{\infty}(J)$, and let (7) be satisfied. Suppose that there exist real numbers $R_{1}, R_{2}$ such that $R_{1} \leq \sigma_{i}^{\prime}(t) \leq R_{2}$ for each $t \in J, i=1,2$, conditions (9), (10) are satisfied for a.e. $t \in J$ and condition (11) is satisfied for all $x \in\left[c_{1}, c_{2}\right]$, where $c_{1}=(b-a) R_{1}+\min \left\{\sigma_{2}(t): t \in J\right\}$ and $c_{2}=(b-a) R_{2}+\max \left\{\sigma_{1}(t): t \in J\right\}$. Then problem $(1),(k), k \in\{3,4\}$, has at least one solution satisfying (13). 
COROLlary 6. Let $f$ be nonincreasing in the second variable $x$. Then, in Theorem 5, conditions (9), (10) can be omitted and (11) can be replaced by (14).

Example 7. If we choose $f, J, \sigma_{1}, \sigma_{2}, R_{1}, R_{2}$ as in Example 3 , then the conditions of Theorem 5 are satisfied for $|\alpha|>1$.

EXAmple 8. If we choose $f, J, \sigma_{1}, \sigma_{2}, R_{1}, R_{2}$ as in Example 4, then the conditions of Corollary 6 are satisfied for $|\alpha|>\pi(b-a) / 2+1$.

Remark. Let $f$ be bounded (sublinear or linear with appropriately small coefficients) in $x$ and $y$. Further, suppose that there exist upper and lower solutions of problem (1), $(k), k \in\{2,3,4\}$, with $\sigma_{1}^{\prime \prime}, \sigma_{2}^{\prime \prime} \in \mathbf{L}_{\infty}(J)$, and for a.e. $t \in J$,

$$
\begin{array}{ll}
f\left(t, x, \sigma_{2}^{\prime}\right) \geq \sigma_{2}^{\prime \prime} & \text { for all } x \leq \sigma_{2}(t), \\
f\left(t, x, \sigma_{1}^{\prime}\right) \leq \sigma_{1}^{\prime \prime} & \text { for all } x \geq \sigma_{1}(t) .
\end{array}
$$

Then condition (7) is sufficient for the solvability of (1), $(k), k \in\{2,3,4\}$. See Lemma 11 for $f$ bounded and [15] for sublinear and linear cases.

3. Proofs. We will consider a one-parameter system of equations

$$
x^{\prime \prime}=\lambda f^{*}\left(t, x, x^{\prime}, \lambda\right), \quad \lambda \in[0,1],
$$

where $f^{*}: J \times \mathbb{R}^{2} \times[0,1] \rightarrow \mathbb{R}$ satisfies the Carathéodory conditions, i.e.

- $f(\cdot, x, y, \lambda): J \rightarrow \mathbb{R}$ is measurable for all $(x, y, \lambda) \in \mathbb{R}^{2} \times[0,1]$,

- $f(t, \cdot, \cdot, \cdot): \mathbb{R}^{2} \times[0,1] \rightarrow \mathbb{R}$ is continuous for a.e. $t \in J$,

- $\sup \{|f(\cdot, x, y, \lambda)|:|x|+|y|<\varrho, \lambda \in[0,1]\} \in \mathbf{L}(J)$ for any $\varrho \in \mathbb{R}_{+}$.

Let $f^{*}$ be chosen such that $f^{*}(t, x, y, 1)=f(t, x, y)$ on $J \times \mathbb{R}^{2}$. Further, put

$$
f_{0 k}(x)=\frac{1}{b-a} \int_{a}^{b} f^{*}(t, x, 0,0) d t \quad \text { for } k \in\{3,4\}
$$

and

$$
f_{02}(x)=\frac{1}{c_{0}}\left[\frac{1}{b-d} \int_{d}^{b} \int_{a}^{s} f^{*}(\tau, x, 0,0) d \tau d s-\frac{1}{c-a} \int_{a}^{c} \int_{a}^{s} f^{*}(\tau, x, 0,0) d \tau d s\right]
$$

where

In our proofs we exploit the following lemma:

$$
c_{0}=\frac{b+d}{2}-\frac{c+a}{2} .
$$

Lemma 9. Let $k \in\{2,3,4\}$ and suppose there exists an open bounded set $\Omega \subset \mathbf{C}^{1}(J)$ such that: (a) for any $\lambda \in(0,1)$, every solution $u$ of problem (17), ( $k$ ) satisfies $u \notin \partial \Omega$; (b) for any root $x_{0} \in \mathbb{R}$ of the equation $f_{0 k}(x)=0$, the condition $x_{0} \notin \partial \Omega$ holds, where $x_{0}$ is considered as a constant function 
on $J ;(\mathrm{c})$ the Brouwer degree $d\left[f_{0 k}, D, 0\right]$ is not zero, where $D \subset \mathbb{R}$ is the set of constants $c$ such that the constant functions $u(t) \equiv c$ belong to $\Omega$. Then problem (17), (k) has at least one solution in $\bar{\Omega}$.

Proof. For $k=2$ see [13] or [14], for $k \in\{3,4\}$ see [3] or [8].

First we prove the existence for problem $(1),(k), k \in\{2,3,4\}$, provided $f$ is bounded and upper and lower solutions are constants.

Lemma 10. Suppose that there exist $r_{1}, r_{2} \in \mathbb{R}$ and $K \in(0, \infty)$ such that $r_{1} \geq r_{2}$ and for a.e. $t \in J$,

$$
\begin{array}{ll}
f(t, x, 0) \geq 0 & \text { for all } x \leq r_{2}, \\
f(t, x, 0) \leq 0 & \text { for all } x \geq r_{1}
\end{array}
$$

and

$$
\int_{a}^{b}|f(t, x, y)| d t \leq K \quad \text { for all } x, y \in \mathbb{R} .
$$

Then problem (1), $(k), k \in\{2,3,4\}$, has at least one solution $u$ with

$$
r_{2} \leq u\left(t_{u}\right) \leq r_{1}
$$

where $t_{u}$ is a point from $J$.

Proof. For every $m \in \mathbb{N}, m \geq 2$, and $(t, x, y) \in J \times \mathbb{R}^{2}$, set

$$
f_{m}(t, x, y)=\left\{\begin{array}{lc}
f(t, x, y) & \text { for }|y|>2 / m, \\
f(t, x, y)+[f(t, x, 0)-f(t, x, y)] m(2 / m-|y|) & \text { for } 1 / m<|y| \leq 2 / m, \\
f(t, x, 0) & \text { for }|y| \leq 1 / m,
\end{array}\right.
$$

and consider system (17) where

$$
f^{*}(t, x, y, \lambda)=\lambda f_{m}(t, x, y)+(1-\lambda) \frac{r_{1}-x}{\left|r_{1}\right|+|x|} .
$$

Define

$$
\Omega=\left\{x \in \mathbf{C}^{1}(J):\|x\|_{\infty}<r,\left\|x^{\prime}\right\|_{\infty}<K+2(b-a)\right\},
$$

where $r=\max \left\{\left|r_{1}\right|,\left|r_{2}\right|\right\}+1+(b-a) K+2(b-a)^{2}$. Now we use Lemma 9 and first prove that for any $\lambda \in(0,1)$ no solution of $(17),(k), k \in\{2,3,4\}$, belongs to $\partial \Omega$. Suppose that $u$ is a solution of $(17),(k), k \in\{2,3,4\}$, for some $\lambda \in(0,1)$. Put $v(t)=u(t)-r_{1}-1 / m$ and suppose

$$
\min \{v(t): t \in J\}=v\left(t_{0}\right)>0 .
$$

Then there exists an interval $[\alpha, \beta] \subset J$ containing $t_{0}$ with $v(t) \geq 0$ and $\left|v^{\prime}(t)\right| \leq 1 / m$ for all $t \in[\alpha, \beta], v^{\prime}(\alpha) \leq 0, v^{\prime}(\beta) \geq 0$. Then for a.e. $t \in(\alpha, \beta)$ we get

$$
v^{\prime \prime}(t)=u^{\prime \prime}(t)=\lambda f_{m}\left(t, u, u^{\prime}\right)+(1-\lambda) \frac{r_{1}-u}{\left|r_{1}\right|+|u|}<\lambda f(t, u, 0) \leq 0 .
$$


On the other hand, $\int_{\alpha}^{\beta} v^{\prime \prime}(t) d t=v^{\prime}(\beta)-v^{\prime}(\alpha) \geq 0$, a contradiction. Analogously we can get a contradiction if

$$
\max \{u(t): t \in J\}<r_{2}-1 / m .
$$

Thus there exists $t_{u} \in J$ such that

$$
r_{2}-1 / m \leq u\left(t_{u}\right) \leq r_{1}+1 / m .
$$

Now, by integrating (1) from $\bar{t}$ to $t$, where $\bar{t}$ is a zero of $u^{\prime}$, we get $\left|u^{\prime}(t)\right| \leq$ $K+2(b-a)$ for all $t \in J$, and integrating the last inequality from $t_{u}$ to $t$ we get $|u(t)| \leq \max \left\{\left|r_{1}\right|,\left|r_{2}\right|\right\}+1 / m+2(b-a)^{2}+K(b-a)$ for all $t \in J$. Thus $u \notin \partial \Omega$.

Now, consider the function $f_{0 k}$ from Lemma 9 which has for all three cases of $k=2,3,4$ the same form $f_{0 k}(x)=\left(r_{1}-x\right) /\left(\left|r_{1}\right|+|x|\right)$. Since the unique root of the equation $f_{0 k}(x)=0$ is $x_{0}=r_{1}$ and the constant function $x_{0} \notin \partial \Omega$, condition (b) of Lemma 9 is satisfied. Finally, we compute the Brouwer degree $d\left[f_{0 k},(-r, r), 0\right]$. It is equal to \pm 1 , because $f_{0 k}(-r)<0$ and $f_{0 k}(r)>0$. Therefore problem (17), $(k), k \in\{2,3,4\}$, has for $\lambda=1, m \geq 2$, at least one solution $u_{m} \in \bar{\Omega}$. Following these considerations for each $m \in \mathbb{N}$, $m \geq 2$, we get a sequence $\left(u_{m}\right)_{m=2}^{\infty}$ of solutions of the problems

$$
x^{\prime \prime}=f_{m}\left(t, x, x^{\prime}\right),(k), \quad k \in\{2,3,4\} .
$$

This sequence is equi-continuous and bounded in $\mathbf{C}^{1}(J)$ and therefore, by the Arzelà-Ascoli Theorem, we can choose a subsequence converging in $\mathbf{C}^{1}(J)$ to $u_{0}$ which is a solution of $(17),(k), k \in\{2,3,4\}$. Moreover, there exists a sequence $\left(t_{u_{m}}\right)_{m=2}^{\infty}$ of points with the property

$$
r_{2}-1 / m \leq u_{m}\left(t_{u_{m}}\right) \leq r_{1}+1 / m,
$$

which implies the existence of $\tau \in J$ such that $r_{2} \leq u_{0}(\tau) \leq r_{1}$.

The second step consists in the change of constant upper and lower solutions to functions depending on $t$.

LEMma 11. Let $\sigma_{1}, \sigma_{2}$ be lower and upper solutions of (1), $(k), k \in$ $\{2,3,4\}$, with $\sigma_{1}^{\prime \prime}, \sigma_{2}^{\prime \prime} \in \mathbf{L}_{\infty}(J)$. Suppose that conditions (7) and (16) are fulfilled. Further, suppose there exists $K \in(0, \infty)$ such that

$$
\int_{a}^{b}|f(t, x, y)| d t \leq K \quad \text { for all } x, y \in \mathbb{R} .
$$

Then problem (1), $(k), k \in\{2,3,4\}$, has at least one solution u satisfying (13). 
Proof. For $n \in \mathbb{N}$ and $(t, x, y) \in J \times \mathbb{R}^{2}$ set

$$
f_{n}(t, x, y)= \begin{cases}f(t, x, y)+(-1)^{i} / n & \text { for }\left|y-\sigma_{i}^{\prime}(t)\right|>2 / n, \\ f(t, x, y)+(-1)^{i} / n+\kappa_{i, n} & \text { for } 1 / n<\left|y-\sigma_{i}^{\prime}(t)\right| \leq 2 / n \\ f\left(t, x, \sigma_{i}^{\prime}(t)\right)+(-1)^{i} / n & \text { for }\left|y-\sigma_{i}^{\prime}(t)\right| \leq 1 / n\end{cases}
$$

where

$$
\kappa_{i, n}=\left[f\left(t, x, \sigma_{i}^{\prime}(t)\right)-f(t, x, y)\right] n\left(2 / n-\left|y-\sigma_{i}^{\prime}(t)\right|\right), \quad i=1,2,
$$

and

$$
g_{n}(t, x, y)= \begin{cases}f_{n}\left(t, x, \sigma_{1}^{\prime}\right)+\frac{A+1 / n-x}{x-A}\left\|\sigma_{1}^{\prime \prime}\right\|_{\infty} & \text { for } x \geq A+1 / n, \\ f_{n}(t, x, y)+w_{1, n} & \text { for } A<x<A+1 / n, \\ f_{n}(t, x, y) & \text { for }-A \leq x \leq A, \\ f_{n}(t, x, y)-w_{2, n} & \text { for }-A-1 / n<x<-A, \\ f_{n}\left(t, x, \sigma_{2}^{\prime}\right)+\frac{A+1 / n+x}{A+x}\left\|\sigma_{2}^{\prime \prime}\right\|_{\infty} & \text { for } x \leq-A-1 / n,\end{cases}
$$

where

$$
\begin{aligned}
w_{i, n} & =\left[f_{n}\left(t, x, \sigma_{i}^{\prime}(t)\right)-f_{n}(t, x, y)\right] n\left(x+(-1)^{i} A\right), \quad i=1,2, \\
A & =(b-a) L+\frac{1}{n}+\max \left\{\left\|\sigma_{1}\right\|_{\infty},\left\|\sigma_{2}\right\|_{\infty}\right\}, \\
L & =K+\frac{b-a}{n}+2(b-a) \max \left\{\left\|\sigma_{1}^{\prime \prime}\right\|_{\infty},\left\|\sigma_{2}^{\prime \prime}\right\|_{\infty}\right\} .
\end{aligned}
$$

We can easily see that $w_{i, n} \equiv 0$ for $\left|y-\sigma_{i}^{\prime}(t)\right| \leq 1 / n$. Now consider the problem

$$
x^{\prime \prime}=g_{n}\left(t, x, x^{\prime}\right),(k), \quad k \in\{2,3,4\} .
$$

We can check that for $r_{1}=2 A+1 / n$ and $r_{2}=-2 A-1 / n$ the function $g_{n}$ satisfies all assumptions of Lemma 10, so problem (18) has a solution $u_{n}$ for each $n \in \mathbb{N}$. Now, using similar arguments to the proof of Lemma 10, we can prove that there exist $t_{n}=t_{n}\left(u_{n}\right) \in J$ such that

$$
-1 / n+\sigma_{2}\left(t_{n}\right) \leq u_{n}\left(t_{n}\right) \leq \sigma_{1}\left(t_{n}\right)+1 / n .
$$

Indeed, putting $v(t)=u_{n}(t)-\sigma_{1}(t)-1 / n\left(\right.$ or $\left.v(t)=\sigma_{2}(t)-u_{n}(t)-1 / n\right)$ and supposing $\min \{v(t): t \in J\}>0$ (or $\max \{v(t): t \in J\}<0$ ), we get a contradiction. We can also easily check that

$$
\int_{a}^{b}\left|g_{n}(t, x, y)\right| d t \leq L \quad \text { for all } x, y \in \mathbb{R} .
$$

From the latter inequality we get $\left\|u_{n}^{\prime}\right\|_{\infty} \leq L$ and $\left\|u_{n}\right\|_{\infty} \leq A$, so $u_{n}$ is a solution of the problem

$$
x^{\prime \prime}=f_{n}\left(t, x, x^{\prime}\right),(k), \quad k \in\{2,3,4\} .
$$


Using the Arzelà-Ascoli Theorem for the sequence $\left(u_{n}\right)_{n=1}^{\infty}$ in the space $\mathbf{C}^{1}(J)$, by a limiting process we obtain a solution $u$ of problem $(1),(k)$, $k \in\{2,3,4\}$, satisfying (13).

Proof of Theorem 1. Suppose that $R_{1}<R_{3}$ and $R_{2}>R_{4}$. Then for sufficiently large $n_{0} \in \mathbb{N}$ we have $R_{4}+2 / n_{0}<R_{2}$ and $R_{3}-2 / n_{0}>R_{1}$. Suppose that $n \in \mathbb{N}, n \geq n_{0}$ and put $r_{1}=\max \left\{\sigma_{1}(t): t \in J\right\}, r_{2}=$ $\min \left\{\sigma_{2}(t): t \in J\right\}$

$$
\begin{array}{r}
g(t, x, y)= \begin{cases}f\left(t, r_{1}+R_{2}(b-a), y\right) & \text { for } x>r_{1}+R_{2}(b-a), \\
f(t, x, y) \quad \text { for } r_{2}+R_{1}(b-a) \leq x \leq r_{1}+R_{2}(b-a), & \text { for } x<r_{2}+R_{1}(b-a), \\
f\left(t, r_{2}+R_{1}(b-a), y\right) & \text { for } y \geq R_{2},\end{cases} \\
h_{n}(t, x, y)= \begin{cases}g\left(t, x, R_{2}\right) & \text { for } R_{4}+2 / n \leq y<R_{2}, \\
g(t, x, y) & \text { for } R_{4}+1 / n<y<R_{4}+2 / n, \\
g\left(t, x, R_{4}+2 / n\right)+w_{4} & \text { for } R_{4}<y \leq R_{4}+1 / n, \\
g\left(t, x, R_{4}\right) & \text { for } R_{3} \leq y \leq R_{4}, \\
g(t, x, y) & \text { for } R_{3}-1 / n \leq y<R_{3}, \\
g\left(t, x, R_{3}\right) & \text { for } R_{3}-2 / n<y<R_{3}-1 / n, \\
g\left(t, x, R_{3}-2 / n\right)-w_{3} & \text { for } R_{1}<y \leq R_{3}-2 / n \\
g(t, x, y) & \text { for } R_{1} \geq y, \\
g\left(t, x, R_{1}\right) & \end{cases}
\end{array}
$$

where

$$
\begin{aligned}
& w_{3}=\left[g\left(t, x, R_{3}-2 / n\right)-g\left(t, x, R_{3}\right)\right] n\left(y-R_{3}+2 / n\right), \\
& w_{4}=\left[g\left(t, x, R_{4}+2 / n\right)-g\left(t, x, R_{4}\right)\right] n\left(y-R_{4}-2 / n\right) .
\end{aligned}
$$

Then for all $x, y \in \mathbb{R}$,

$$
\int_{a}^{b}\left|h_{n}(t, x, y)\right| d t \leq \int_{a}^{b} h(t) d t=K,
$$

where $h(t)=\sup \left\{\left|h_{n}(t, x, y)\right|: x \in\left[r_{2}+R_{1}(b-a), r_{1}+R_{2}(b-a)\right], y \in\right.$ $\left.\left[R_{1}, R_{2}\right]\right\}$. We can see that $h_{n}$ satisfies the conditions of Lemma 11 and so the problem

$$
x^{\prime \prime}=h_{n}\left(t, x, x^{\prime}\right),
$$

has a solution $u_{n}$ satisfying (13).

Let us prove a priori estimates for $u_{n}^{\prime}$. It follows from (2) that there exist $a_{0} \in(a, c)$ and $b_{0} \in(d, b)$ with $u_{n}^{\prime}\left(a_{0}\right)=u_{n}^{\prime}\left(b_{0}\right)=0$. Suppose that $\max \left\{u_{n}^{\prime}(t): t \in\left[a, b_{0}\right]\right\}=u_{n}^{\prime}(\gamma)>R_{2}+1 / n$. Then $\gamma \neq b_{0}$ and there exists $(\alpha, \beta) \subset\left(a, b_{0}\right)$ such that $u_{n}^{\prime}(\beta)=R_{2}, u_{n}^{\prime}(\alpha)=R_{2}+1 / n$ and $R_{2} \leq u_{n}^{\prime}(t) \leq$ $R_{2}+1 / n$ for all $t \in(\alpha, \beta)$. Thus $\int_{\alpha}^{\beta} u_{n}^{\prime \prime}(t) d t=-1 / n<0$. On the other hand, 
by (11), (12) and the construction of $h_{n}$,

$$
\int_{\alpha}^{\beta} u_{n}^{\prime \prime}(t) d t=\int_{\alpha}^{\beta} g\left(t, u_{n}, R_{2}\right) d t \geq 0,
$$

a contradiction. Similarly, supposing $\min \left\{u_{n}^{\prime}(t): t \in\left[a, b_{0}\right]\right\}<R_{1}-1 / n$, we get a contradiction. Therefore

$$
R_{1}-1 \leq R_{1}-1 / n \leq u_{n}^{\prime}(t) \leq R_{2}+1 / n \leq R_{2}+1
$$

for all $t \in\left[a, b_{0}\right]$.

Now, suppose that $\max \left\{u_{n}^{\prime}(t): t \in\left[b_{0}, b\right]\right\}=u_{n}^{\prime}(\gamma)>R_{4}+1 / n$. Then $\gamma \in\left(b_{0}, b\right]$ and there exists $(\alpha, \beta) \subset\left(b_{0}, b\right)$ such that $u_{n}^{\prime}(\alpha)=R_{4}, u_{n}^{\prime}(\beta)=$ $R_{4}+1 / n$, and $R_{4} \leq u_{n}^{\prime}(t) \leq R_{4}+1 / n$ for all $t \in(\alpha, \beta)$. Thus $\int_{\alpha}^{\beta} u_{n}^{\prime \prime}(t) d t=$ $1 / n>0$. On the other hand, by (11), (12) and the construction of $h_{n}$,

$$
\int_{\alpha}^{\beta} u_{n}^{\prime \prime}(t) d t=\int_{\alpha}^{\beta} g\left(t, u_{n}, R_{4}\right) d t \leq 0,
$$

a contradiction. Similarly, supposing $\min \left\{u_{n}^{\prime}(t): t \in\left[b_{0}, b\right]\right\}<R_{3}-1 / n$, we get a contradiction. Thus, by (21),

$$
R_{1}-1 / n \leq u_{n}^{\prime}(t) \leq R_{2}+1 / n
$$

for all $t \in J$. Integrating (22) from $t_{u}$ to $t$ and using (13), we get

$$
r_{2}+R_{1}(b-a)-\frac{b-a}{n} \leq u_{n}(t) \leq r_{1}+R_{2}(b-a)+\frac{b-a}{n}
$$

for all $t \in J$.

For each $n \in \mathbb{N}, n \geq n_{0}$, we have a solution $u_{n}$ satisfying estimates (22) and (23). Since the sequence $\left(u_{n}\right)_{n=n_{0}}^{\infty}$ is bounded and equi-continuous in $\mathbf{C}^{1}(J)$, we can use the Arzelà-Ascoli Theorem and get a solution $u$ of the problem

$$
x^{\prime \prime}=g\left(t, x, x^{\prime}\right),(2),
$$

with $r_{2}+R_{1}(b-a) \leq u(t) \leq r_{1}+R_{2}(b-a)$ and $R_{1} \leq u^{\prime}(t) \leq R_{2}$ for all $t \in J$. Hence $u$ is a solution of (1), (2) as well.

Proof of Theorem 5. We can follow the previous proof and make some simplifications. E.g. instead of $h_{n}$ we use

$$
h(t, x, y)= \begin{cases}g\left(t, x, R_{2}\right) & \text { for } y>R_{2}, \\ g(t, x, y) & \text { for } R_{1} \leq y \leq R_{2}, \\ g\left(t, x, R_{1}\right) & \text { for } y<R_{1}\end{cases}
$$

and for a priori estimates of $u^{\prime}$ we only need condition (11), because in the case of problem (1), (3), $u^{\prime}$ has zero values at the end points, and if we consider periodic problem (1), (4), it is sufficient to prove the estimate of $u^{\prime}$ only at one of the end points. 


\section{References}

[1] S. R. Bernfeld and V. Lakshmikantham, An Introduction to Nonlinear Boundary Value Problems, Academic Press, New York, 1974.

[2] E. N. Dancer, On the ranges of certain damped nonlinear differential equations, Ann. Mat. Pura Appl. (4) 119 (1979), 281-295.

[3] R. Gaines and J. Mawhin, Coincidence Degree and Nonlinear Differential Equations, Lecture Notes in Math. 568, Springer, Berlin, 1977.

[4] W. Gao and J. Wang, On a nonlinear second order periodic boundary value problem with Carathéodory functions, Ann. Polon. Math. 62 (1995), 283-291.

[5] I. T. Kiguradze, Some Singular Boundary Value Problems for Ordinary Differential Equations, Univ. Press, Tbilisi, 1975 (in Russian).

[6] H. W. Knobloch, Eine neue Methode zur Approximation periodischer Lösungen nicht-linearer Differentialgleichungen zweiter Ordnung, Math. Z. 82 (1963), 177197.

[7] A. Lepin and L. Lepin, Boundary Value Problems for Ordinary Differential Equations of the Second Order, Zinatne, Riga, 1988 (in Russian).

[8] J. Mawhin, Topological Degree Methods in Nonlinear BVPs, CBMS Regional Conf. Ser. in Math. 40, Providence, R.I., 1979.

[9] J. Mawhin and J. R. Ward Jr., Periodic solutions of some forced Liénard differential equations at resonance, Arch. Math. (Basel) 41 (1983), 337-351.

[10] J. J. Nieto, Nonlinear second order periodic boundary value problems, J. Math. Anal. Appl. 130 (1988), 22-29.

[11] P. Omari, Non-ordered lower and upper solutions and solvability of the periodic problem for the Liénard and the Rayleigh equations, Rend. Inst. Mat. Univ. Trieste 20 (1988), 54-64.

[12] I. Rachůnková, The first kind periodic solutions of differential equations of the second order, Math. Slovaca 39 (1989), 407-415.

[13] - An existence theorem of the Leray-Schauder type for four-point boundary value problems, Acta Univ. Palack. Olomuc. Fac. Rerum Nat. 100, Math. 30 (1991), 4959.

[14] -, On a transmission problem, ibid. 105, Math. 31 (1992), 45-59.

[15] - On resonance problems for the second order differential equations, preprint.

[16] R. Reissig, Schwingungssätze für die verallgemeinerte Liénardsche Differentialgleichung, Abh. Math. Sem. Univ. Hamburg 44 (1975), 45-51.

[17] N. I. Vasil'ev and Yu. A. Klokov, Foundations of the Theory of Boundary Value Problems for Ordinary Differential Equations, Zinatne, Riga, 1978 (in Russian).

[18] F. Zanolin, Periodic solutions for differential systems of Rayleigh type, Rend. Inst. Mat. Univ. Trieste 12 (1980), 69-77.

Department of Mathematics

Palacký University, Tomkova 40

77900 Olomouc, Czech Republic

E-mail: rachunko@risc.upol.cz 